\title{
Predictive value of spleen stiffness in hepatocellular carcinoma
}

\author{
Matthias Buechter ${ }^{1,2}$, Alisan Kahraman ${ }^{1}$ \\ ${ }^{1}$ Department of Gastroenterology and Hepatology, University Clinic of Essen, University of Duisburg-Essen, Essen, Germany; ${ }^{2}$ St. Nikolaus- \\ Stiftshospital, Andernach, Germany \\ Correspondence to: Matthias Buechter. Department of Gastroenterology and Hepatology, University Clinic of Essen, University of Duisburg-Essen, \\ Essen, Germany. Email: Matthias.Buechter@uk-essen.de.
}

Submitted Aug 13, 2019. Accepted for publication Sep 05, 2019.

doi: $10.21037 /$ hbsn.2019.09.05

View this article at: http://dx.doi.org/10.21037/hbsn.2019.09.05

In their Letter to the Editor, Wei et al. address on the role of spleen stiffness measurement (SSM) for predicting the late recurrence ( $>24$ months post-surgery) of hepatocellular carcinoma (HCC) after resection (1). They comment on a recently published manuscript by Marasco and colleagues, who could show that spleen stiffness measured by transient elastography was the only predictor of late HCC recurrence after surgery (2).

Portal hypertension $(\mathrm{PH})$ is a major complication of liver cirrhosis and the most prominent contributing factor for decompensating events such as variceal bleeding. Measurement of the hepatic venous pressure gradient (HVPG) is the gold standard for PH assessment. However, the clinical relevance of HVPG is limited by its invasiveness and the resulting restriction to tertiary centers in many countries. Therefore, non-invasive tools to assess $\mathrm{PH}$ and its severity have become methods of interest during the last decades. Hereof, several studies indicate that SSM is able to noninvasively reflect changes in $\mathrm{PH}$ occurring in advanced stages of chronic liver disease (CLD). It is strongly correlated with presence and degree of esophageal and gastric varices, risk of variceal bleeding, and actually HVPG (3). The pathophysiology regarding alterations in spleen stiffness seems to be multifactorially: (I) firstly, it can be partly ascribed to increased splanchnic circulation occurring in advanced stages of cirrhosis; (II) in addition, disordered spleen immunity, e.g., invasion of immune cells, plays an important role in the dynamic process of spleen fibrosis (4).

Similar to PH, HCC development plays an important role in patients with CLD increasing morbidity and mortality. Liver resection is the treatment of choice among these patients as long as solitary tumors are present and the functional reserve is sufficient. However, HCC recurrence rates are reported to occur in up to $70 \%$ at 5 years. Distinction between early ( $<24$ months) and late HCC recurrence ( $>24$ months) is proposed, while the latter is considered as de novo tumor. In contrast to early HCC recurrence where predictive factors are well established (e.g., tumor size, tumor number), only poor data are available for the prediction of late recurrence (5).

In their cohort, Marasco et al. included 157 patients with a first HCC diagnosis who underwent curative hepatic resection according to AASLD guidelines. Out of 87 patients who were included in the late recurrence group, 27 developed HCC. Late HCC recurrence was associated with the presence of esophageal varices, spleen size, and liver and spleen stiffness, respectively. However, multivariate analysis showed that only SSM was an independent predictor of late HCC recurrence (optimal cut-off value $70 \mathrm{kPa}$, positive predictive value and negative predictive value of $75 \%$, $\mathrm{P}<0.0002)(2)$.

It is widely accepted that $\mathrm{PH}$ is the main driver of complications among patients with liver cirrhosis, including the occurrence of HCC. The positive correlation between (the degree of) $\mathrm{PH}$, carcinogenesis and thus HCC development was pointed out in different publications. Conversely, reduction of portal pressure by insertion of transjugular intrahepatic portosystemic shunt (TIPS) is significantly associated with reduced rates of de novo HCC (6). The results of Marasco and colleagues are therefore not exceedingly surprising: severity of liver fibrosis or cirrhosis, mirrored by spleen stiffness as indicator of $\mathrm{PH}$, is closely related to HCC development. The authors therefore suggest performing SSM before HCC resection, in order to identify patients with high risk for HCC recurrence $(\mathrm{SSM}>70 \mathrm{kPa})$ and enable clinicians to provide 
a more individualized surveillance program.

However, some limitations of SSM as predictor for HCC should be mentioned. Firstly, there is solid evidence supporting the fact that HCC occurs without presence of cirrhosis and PH. Apart from chronic hepatitis B, patients suffering from non-alcoholic steatohepatitis are at risk for HCC development without presence of advanced fibrosis (7). Furthermore, technical and anatomical limitations sometimes hamper the applicability of SSM (spleen diameter, presence of ascites, obese patients). Valid measurements are therefore not obtainable among all patients.

In conclusion, the results of Marasco et al. once again impressively illustrate the predictive power of SSM: it seems to be a confirmable non-invasive tool for monitoring CLD and identifying patients at high risk for PH-related complications.

\section{Acknowledgments}

None.

\section{Footnote}

Conflicts of Interest: The authors have no conflicts of interest to declare.

Ethical Statement: The authors are accountable for all aspects of the work in ensuring that questions related to the accuracy or integrity of any part of the work are appropriately investigated and resolved.

\section{References}

1. Wei W, Li L, Kong G, et al. Spleen in hepatocellular carcinoma: More complexity and importance than we knew. J Hepatol 2019;70:805-6.

2. Marasco G, Colecchia A, Colli A, et al. Role of liver and spleen stiffness in predicting the recurrence of hepatocellular carcinoma after resection. J Hepatol 2019;70:440-8.

3. Buechter M, Manka P, Theysohn JM, et al. Spleen stiffness is positively correlated with HVPG and decreases significantly after TIPS implantation. Dig Liver Dis 2018;50:54-60.

4. Kondo R, Kage M, Iijima H, et al. Pathological findings that contribute to tissue stiffness in the spleen of liver cirrhosis patients. Hepatol Res 2018;48:1000-7.

5. Heimbach JK, Kulik LM, Finn RS, et al. AASLD guidelines for the treatment of hepatocellular carcinoma. Hepatology 2018;67:358-80.

6. Hüsing-Kabar A, Meister T, Köhler M, et al. Is de novo hepatocellular carcinoma after transjugular intrahepatic portosystemic shunt increased? United European Gastroenterol J 2018;6:413-21.

7. Ertle J, Dechêne A, Sowa JP, et al. Non-alcoholic fatty liver disease progresses to hepatocellular carcinoma in the absence of apparent cirrhosis. Int J Cancer 2011;128:2436-43.
Cite this article as: Buechter M, Kahraman A. Predictive value of spleen stiffness in hepatocellular carcinoma. Hepatobiliary Surg Nutr 2020;9(1):101-102. doi: 10.21037/hbsn.2019.09.05 